\title{
Cost-effective microfabrication of sub-micron- depth channels by Femto-laser anti-stiction texturing
}

\author{
Shadi Karimi ${ }^{1}$, Pouya Mehrdel ${ }^{1}$, Jasmina Casals-Terré ${ }^{1}$, Josep Farré-Llados ${ }^{1}$ \\ ${ }^{1}$ Mechanical, Fluids and Aerospace Engineering, Universidad Politécnica de Cataluña, Terrassa, \\ Barcelona, Spain
}

E-mail: josep.farre.1lados@upc.edu

Received $\operatorname{xxxxxx}$

Accepted for publication $\mathrm{xxxxxx}$

Published xxxxxx

\begin{abstract}
Micro Electro Mechanical Systems (MEMS) and microfluidic devices have found numerous applications in the industrial sector. However, they require a fast, cost-effective and reliable manufacturing process in order to compete with the conventional methods. Particularly, at the sub-micron scale, the manufacturing od devices are limited by the dimensional complexity. A proper bonding and stiction prevention of these sub-micron channels are two of the main challenges faced during the fabrication process of low aspect ratio channels. Especially, in case of using flexible materials such as polydimethylsiloxane (PDMS). This study presents a direct laser microfabrication method of sub-micron channels using an infrared (IR) ultrashort pulse (femtoseconds) capable of manufacturing extremely low aspect ratio channels. These microchannels are manufactured and tested varying their depth from $0.5 \mu \mathrm{m}$ to $2 \mu \mathrm{m}$ and width of $15,20,25$, and $30 \mu \mathrm{m}$. The roughness of each pattern was measured by an interferometric microscope. Additionally, the static contact angle of each depth was studied to evaluate the influence of femtosecond laser fabrication method on the wettability of the glass substrate. PDMS, which is a biocompatible polymer, was used to provide a watertight property to the sub-micron channels and also to assist the assembly of external microfluidic hose connections. A 750nm depth watertight channel was built using this methodology and successfully used as a blood plasma separator (BPS).

The device was able to achieve $100 \%$ pure plasma without stiction of the PDMS layer to the sub-micron channel within an adequate time. This method provides a novel manufacturing approach useful for various applications such as point-of-care devices.
\end{abstract}

Keywords: Femtolaser, Biofabrication, Microfluidic, Blood/plasma separation

\section{Introduction}

The fast improvement of MEMS and microfluidics during the last decade has allowed the development of new research areas [1][2]. Currently, this research has consolidated on new devices that require cost-effective and reliable manufacturing processes. Rapid prototyping is an important stage in the fabrication of these systems, enabling fast verification of designs without the need for microfluidic simulations that do not always prove to be accurate enough [3]. For these applications, there are many materials and manufacturing methods such as photo sensible resins (SU-8) and polymers (PDMS) which are shaped by conventional soft lithography or 
selective laser sintering printers [4]-[9]. PDMS is one of the most used polymers for its excellent optical properties and compatibility with different microfluidics and biological applications, however, its low Young modulus can produce a collapse of the structure at low aspect ratios. Glass is another candidate but the hose connections can not be easily integrated. For diagnostic applications, there is a need to achieve low aspect ratio channels which can be connected easily to external pipes. Structuring adequately the surface of the glass can allow the combination of both materials for low aspect ratio structures and facilitating the fabrication of biocompatible applications.

Blood is classified as connective tissue and is composed of two elements: plasma and blood cells. Plasma is essentially an aqueous solution and constitutes around $55 \%$ of the blood volume. Blood cells are Red Blood Cells (RBCs), White Blood Cells (WBCs), and platelets. Plasma is the base of a wide range of assays and is needed at clinical chemistry testing for prognosis [8]. To this end, there is a need for a low cost and reliable method to produce blood plasma filters that can separate plasma from a single droplet of blood. Previous researchers have focused on low aspect ratio channels to achieve filtration using wet chemical etching on the glass [10][11]. This method is based on extremely aggressive chemicals with a huge environmental impact and difficult to industrialize. Therefore, there is a manufacturing limitation to provide cost-effective and reliable method channels with a characteristic height lower than $1.5 \mu \mathrm{m}$ for blood-plasma filtering. RBCs are biconcave, measuring approximately 6 to $8 \mu \mathrm{m}$ in diameter and a thickness at the thickest point of 2 to $2.5 \mu \mathrm{m}$ and a minimum thickness in the center of 0.8 to $1 \mu \mathrm{m}$, being much smaller than most other human cells which under high shear flows the shape of the red blood cells can deform [12] reducing its smaller dimension even to less than $1 \mu \mathrm{m}$. Consequently, channels with less than one micron in depth are required to achieve a reliable filtration rate and also it is necessary to avoid high shear stress to prevent cell fragmentation.

There are a large portfolio of manufacturing methods to produce channel dimension in micron-scale [13]-[15], however, at the sub-micron range, the manufacturing processes are more complex [16] and limited, especially if watertight channels are required [17]. Focusing on watertight channels, Chen et. al [18] presented a fabrication method using PDMS that took advantages of simplicity and low cost although the material deformed easily. Turning this disadvantage into an advantage, Park et.al [19] studied the PDMS collapse related to the ratio of width and depth of the original micro-channel to form sub-micron channels afterward. Reactive Ion Etching (RIE) is a well-known manufacturing method capable to manufacture from $40-\mathrm{nm}$ to $300-\mu \mathrm{m}$-depth channels on different base materials such as glass [20] or PDMS [21] but limited to cleanroom facilities.
Laser beam machining (LBM) is a growing non-traditional machining process where laser thermal energy is used to remove material from metallic or non-metallic surfaces [22]. Different laser source wavelengths are used to machine infrared (IR) and ultraviolet (UV) wavelengths range. $\mathrm{CO}_{2}$ IR lasers are the most widely used and more cost-effective compared to UV although when it is used on some polymeric materials the temperature of the laser not only reduces the precision but also changes the material properties. This technology has been used in micron range to produce through holes which requires assembly to achieve the whole microfluidic device, such as Hao-Bing et al. [23] where the channel shape was defined using $\mathrm{CO}_{2}$ laser on a precured film.

Other authors used Polymethylmethacrylate (PMMA) and pressure-sensitive adhesives [3][24][25] but the range of channel thickness was always limited to the micron range and bonding was more complex, therefore different materials were required [24] or different laser passes [26]. From the other hand controlling the wettability of the surface is important for various practical applications. For instance, hydrophilic surfaces are useful for capillary behavior in microfluidic channels while hydrophobic surfaces are good for selfcleaning of contaminations. However, hydrophobic surfaces are difficult to bond. As glass substrates are useful for various applications, we study the controlled modification of characterized surface wettability with femtosecond laser passes [25].

UV laser sources energy concentration is high and can generate a smaller focus spot size in sub-micron level, but the range of materials is limited. Matthew et al. [27] used UV laser ablation to machine micron-sized channel on biocompatible polymers, however further thermal bonding was required. IR femtosecond laser technology joints the wide range of materials with precision. Nowadays, IR laser sources (i.e. ytterbium) with ultrashort pulses opens the possibility to achieve high precision. Ultra-short-pulse lasers can machine substrates at the wavelength where they are usually transparent. This provides a precision down to the tenth of nanometers without affecting the nearby area [28]. Researchers implemented this technology with a directly fully enclosed channel [29] in the micron range, and it is being introduced in the industry to drill high-aspect-ratio holes.

This manuscript presents a laser direct microfabrication method that uses IR femtosecond laser pulses to produce antistiction [30] extremely low aspect ratio sub-micron channels on a glass substrate. At atmospheric conditions, different channel depths has been manufactured from $500 \mathrm{~nm}$ to $2 \mu \mathrm{m}$. The sub-micron channel is sealed by a PDMS layer via Oxygen-plasma to enable the watertight behavior without the need of any other step or layer.

A BPS is manufactured by this method to filter the cellular component of blood and achieves $100 \%$ pure plasma from undiluted blood. This method improves the reproducibility on 
the size and the shape from the method presented by Park et.al [19] which is faster and easier than RIE [20][21]. Importantly, the stiction of the PDMS layer either during the manufacturing process or during the operation [27] is avoided due to the texture and roughness of the laser machined surface of the glass. To confirm if surface wettability is a function of surface morphology, surface roughness is measured and the regime is studied between two well-known wetting theories by Wenzel [31] and Cassi-Baxter [32].

\section{Materials and method}

Firstly the potential of femtosecond laser micromachine for rapid prototyping of microfluidic channels on the surface of glass substrates was assessed. To validate the femtosecond laser manufacturing accuracy in the sub-micron range, a template with different channels was designed. Each channel was formed by two parallel rectangular blocks to investigate the in-plane accuracy. The distance between the two blocks (channel width) is the same as the width of the block. Different channel depth was studied, modified $\mathrm{H}$ parameter from 500 $\mathrm{nm}$ to $2 \mu \mathrm{m}$ as shown in Figure 1. This figure shows the details of dimension $\mathrm{W}$ that characterizes these columns which vary from $15 \mu \mathrm{m}$ to $30 \mu \mathrm{m}$. These rectangular cross-section microchannels were fabricated by performing different laser passes for each depth with the same laser power.

a)
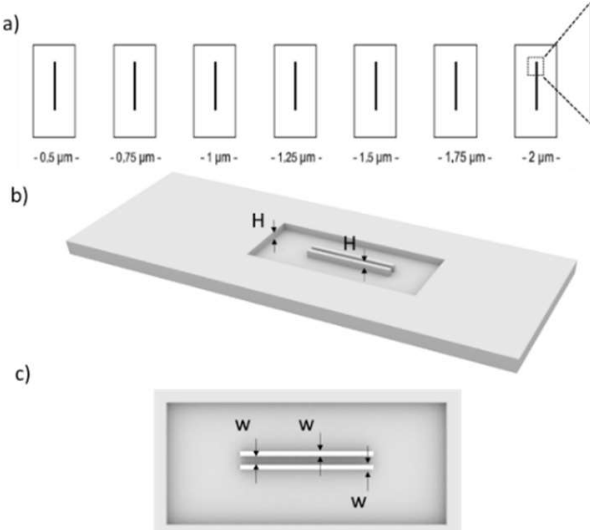

Figure 1: (a) 2D view of the seven different Template in depth $(H)$ from the range of $0.5 \mu m$ (Nanochannels) to $2 \mu \mathrm{m}$ (microchannels). Each template fabricated in four different channel width (w): $15 \mu \mathrm{m}, 20 \mu \mathrm{m}, 25 \mu \mathrm{m}$, and $30 \mu \mathrm{m}$. (b) the 3-D view schematic of one template. (c) the top view of the template.

After studying the influence of different patterns and different height on the glass, the desired sketch was carved to develop a blood plasma filtering device. This pattern is a reservoir with an array of pillars which not only holds the weight of PDMS on the top but also conduct the extracted plasma in the reservoir to the plasma collector channels as it is shown in Figure 2.
To manufacture the desired shape and depth on the glass, the femtosecond laser works as an engraving machine using a laser spot of approximately $10 \mu \mathrm{m}$, with the power of $20 \mathrm{~W}$, and pulse duration of approximately $350 \mathrm{fs}$. The femtoseconds laser is controlled by a CNC system and depending on the depth different passes are required on the surface. Each laser pass is $120^{\circ}$ tilt from its previous to ensure the maximum uniformity at the engraving area. First laser pass made 0.5$\mu \mathrm{m}$-depth and after that for each laser pass, $0.25-\mu \mathrm{m}$-depth is used. Therefore, for the channel of $2 \mu \mathrm{m}$ depth, the laser performs 7 passes.

Surface roughness has a strong effect on wettability of a surface and the contact angle. The effect of roughness changes if the droplet wets the surface grooves or if air pockets are left between the droplet and the surface. Two different theories were developed to explain the effect of surface roughness on wettability. If the surface is wetted homogeneously, the droplet is in Wenzel [31] state. In Wenzel state, adding surface roughness will enhance the wettability caused by the chemistry of the surface. This model predicts enhanced hydrophilicity for hydrophilic surfaces and an enhanced hydrophobicity for hydrophobic surfaces. This model takes into account a complete wetting of droplet with the underneath substrate. If the surface is wetted heterogeneously, the droplet is in Cassie-Baxter [32] state. This model assumes that the liquid does not completely wet the roughened substrate. The Cassie-Baxter model predicts that roughening a surface always increases the contact angle. If a surface is rough enough so that air may be entrapped between the liquid and the solid, the interface becomes composite and the contact angle increases with roughness even if the surface chemistry is intrinsically hydrophilic. The most stable contact angle can be connected to the Young contact angle. The contact angles calculated from the Wenzel and Cassie-Baxter equations are

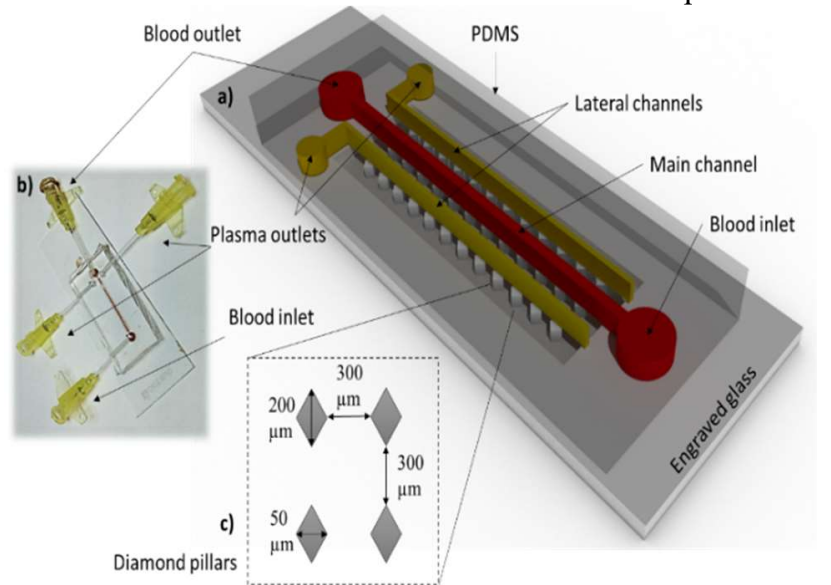

Figure 2: (a) Schematic of the BPS device which contains a PDMS block on the top part and an engraved glass by laser on the bottom part. (b) a picture of the real device. (c) The pattern that is carved on the glass with the dimensions of the array of diamond pillars. 
good approximations of the most stable contact angles with real surfaces.

The obtained nano and microchannels were measured using a confocal microscope model Sensofar PLU neox with a 10XDI objective to evaluate the accuracy on the sub-micron range depth. Furthermore, the surface roughness was evaluated using an optical zoom $4 \mathrm{X}$ in an area of $317.06 \times 237.38 \mu \mathrm{m}^{2}$. The channel template of Figure 1a was manufactured three times. This template contains four different width columns (15 $\mu \mathrm{m}, 20 \mu \mathrm{m}, 25 \mu \mathrm{m}$, and $30 \mu \mathrm{m}$ ) that form a channel between them with the same dimension, see drawing dimension $\mathrm{W}$ at the detail of Figure 1.

In order to investigate the influence of the roughness generated by the femtosecond laser irradiation on surface wettability, the measurement of the static contact angle of each template was carried out using an optical video contact angle system (OCA software, SCA20 module, Dataphysics). To perform the test, a $0.7 \mu \mathrm{L}$ droplet of de-ionized water $25^{\circ}$, having a surface tension of $71.99 \pm 0.05 \mathrm{mN} / \mathrm{m}$, was dispensed on the samples using a $500-\mu \mathrm{L}$ Hamilton syringe unit. The static contact angle based on the Young-Laplace fitting was measured using the sessile drop method with dedicated software.

\subsection{Device Fabrication}

The device is conceived in two parts: A PDMS layer where the microchannels and the external connection are located in a glass holder that has the sub-micron reservoir pattern. Figure 3 shows the three steps of the manufacturing process. Step A includes a conventional soft lithography process to make a mold. The pattern of the microchannels on the PDMS is engraved by this mold. Besides, the syringe pump is connected through the external connection on the PDMS. Step B uses femtoseconds laser pulses to define the low-aspect-ratio pillar shapes and the sub-micron channel walls on the glass. for this manner firstly, a 2D model of the sub-micron channel was defined using AutoCAD 2018 as shown in Figure 2c. The model with Rhinoceros was edited to define the regions to manufacture the micro-channel of a certain depth. A

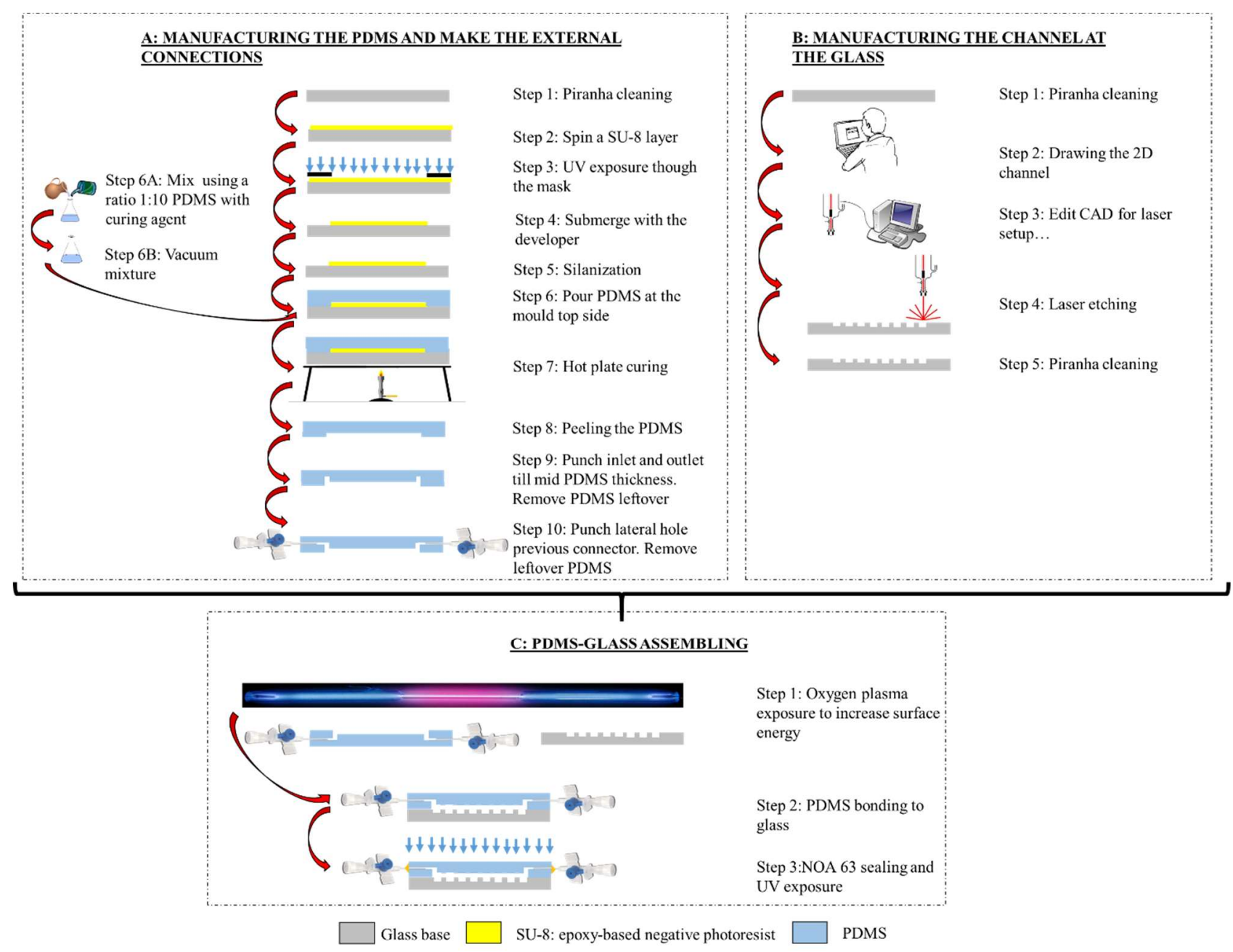

Figure 3: Manufacturing method steps. A is the manufacturing method of the micro-channels on a PDMS base. B is the nanochannels manufacturing method on a glass base. $C$ is the bonding process that has a watertight channel. 
femtosecond laser model AgieCharmilles LASER P $400 \mathrm{U}$ was used. The sub microchannel was cleaned using sonication and immersion in a Piranha solution as following:

First: sonication using acetone for $15 \mathrm{~min}$ and rinsing with distilled water.

Second: 20min in Piranha (1:3 H2O2:H2SO4) and rinsing with distilled water and dry on the hot plate at $95^{\circ} \mathrm{C}$ for 10 minutes right before the PDMS-Glass bonding process starts.

In step $\mathrm{C}$ the PDMS is bonded to the glass via Oxygen plasma. In order to ensue the hose connections are sealed completely, Norland Optical Adhesive 63 (NOA63) is used. This adhesive is a clear, colorless, liquid photopolymer that will cure via UV light exposure.

\subsection{Blood plasma separator (BPS)}

In order to validate the fabricated sub-micron pillars, a BPS system was designed and manufactured as an application. The BPS contains two parts; the bottom part is a glass which carved by laser in depth of $0.75 \mu \mathrm{m}$ and contains an array of diamond pillars in order to hold the weight of the top part which is a PDMS block. The PDMS has three separated channels, one main channel in the middle of two lateral channels. The main channel is filled by the blood sample and two collector channels are designed to collect the extracted plasma by capillary force. The PDMS and the glass were bonded via Oxygen plasma. The PBS device was placed under an optical upright microscope (Dino-Lite Digital Microscope Edge Series USB Cameras).

The blood samples that are used in the BPS were collected from healthy volunteers from Banc de Sang i Teixits de Catalunya.. The samples were stored in a vacutainer collection tube which contains anti-coagulant (Ethylenediamine tetraacetic acid) to postpone the coagulation phenomena during the experimental setup. To maximize the livability of the cellular components, the samples were stored at $4{ }^{\circ} \mathrm{C}$ temperature and were gently mixed back and forward to prevent possible undesirable cell sedimentation prior to testing. All the experiments were processed at the room temperature (20-25 $\left.{ }^{\circ} \mathrm{C}\right)$.

The blood sample was injected from the blood inlet using a syringe pump at a flow rate of $10 \mathrm{ml} / \mathrm{min}$. The height of the pillars is $0.75 \mu \mathrm{m}$ to prevent entrance of red blood cells at the plasma collector channel even at high shear forces. Then pure plasma was driven to the plasma reservoir and blood with a higher concentration of red blood cells was drawn from blood outlet.

To evaluate the quality of the plasma and the performance of the sub-micron filter, the plasma purity parameter was measured. An image processing was developed by an opensource ImageJ software to analyze the particles by their population. To determine the purity of the extracted plasma, a rectangular area $(200 \mu \mathrm{m} \times 400 \mu \mathrm{m})$ in the blood inlet was selected and compared with the same size area in the plasma outlet. These areas were captured by microscope after the plasma collector channels were filled completely. The number of blood cells in these areas were counted by the particle analysis method of the ImageJ software. The plasma purity evaluates the number of red blood cells ratio at the plasma outlets compared to the initial red blood cells at the inlet according to equation 1 .

Purity $\%=\left(1-\left(\frac{\text { final cells density }}{\text { initial cells density }}\right)\right) * 100 \%$

\section{Results \& discussion}

\subsection{Sub-micron channels manufacturing method}

During the manufacturing process, the laser is focused on the material surface that absorbs the energy and transforms it into the gaseous state to generate the sub-micron channel. For extremely low depth $0.5 \mu \mathrm{m}$, the ablation of the surface may not be complete and, according to Figure 4a, some protruding peaks appear. Figure $4 \mathrm{~b}$ shows a cross-section of the manufactured part and in this figure. For $0.5-\mu \mathrm{m}-$ depth channels, the material ablation is not completed or some ablated material is deposited and bonded again at the surface forming some protruding peaks. These peaks avoid the achievement of a flat surface and prevent a complete seal with the PDMS cover. Figure 4 clearly shows the pattern of the
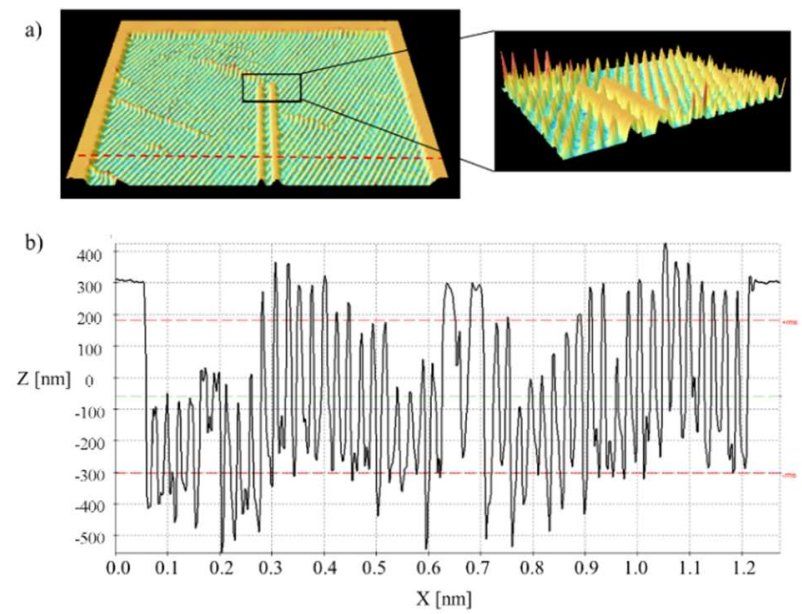

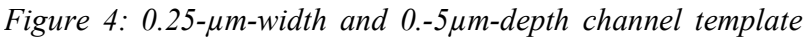
measurement using a confocal microscope. (a) shows the $3 D$ view of the region treated with the laser that contains some protruding regions. Redline is the cross-section position is shown in (b). The cross-section shows the protruding regions are higher in depth than the level of the original surface. 
laser machining direction for $0.5-\mu \mathrm{m}$-depth channels while in $0.75-\mu \mathrm{m}$-depth channels template this pattern disappears as shown in Figure 5. This is due to the fact that two laser passes were performed to define the depth. Furthermore, the
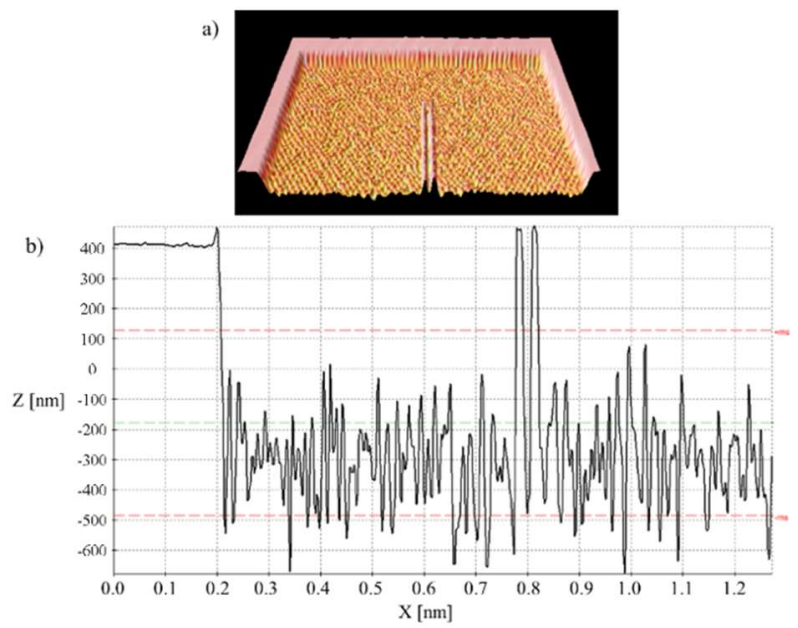

Figure 5: Channel template of $0.75 \mu \mathrm{m}$ depth and $15 \mu \mathrm{m}$ width measurement using a confocal microscope

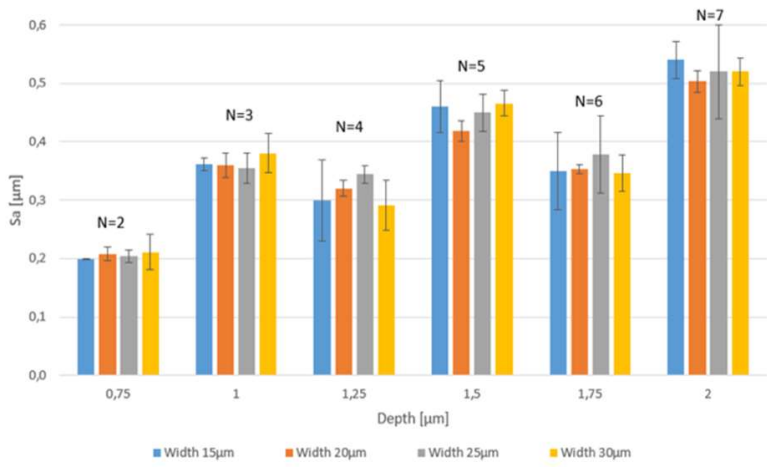

Figure 6: Confocal surface roughness (Sa) measurements from $0.75 \mu \mathrm{m}$ to $2 \mu \mathrm{m}$ depth channel template with a different width. It expresses, as an absolute value, the difference in height of each point compared to the arithmetical mean of the surface. $N$ is the number of laser passes.

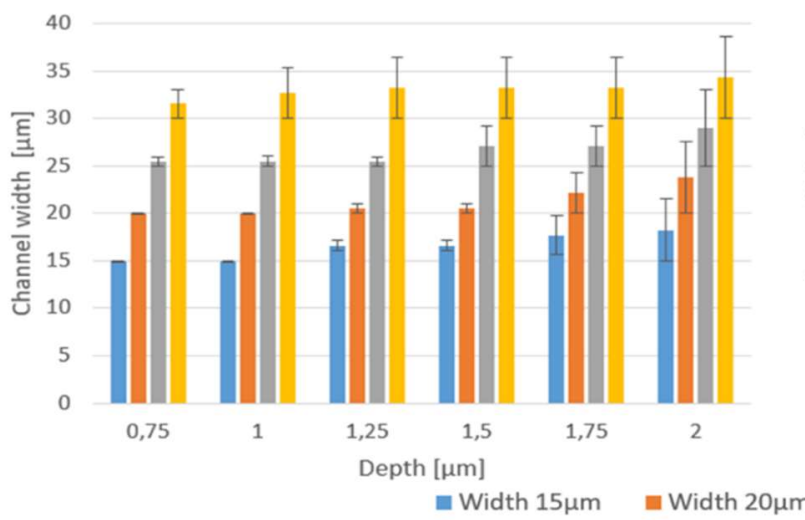

protruding peaks disappear as well, see Figure 5 and more roughness is generated on the surface.

Figure 6 shows that there is no influence of the channel width on the roughness and outlines that the roughness increases as the depth of the channel increases, which means the number of laser passes increases. Additionally, when the number of laser passes is an odd number (the case of $1-\mu \mathrm{m}, 1.5-\mu \mathrm{m}$, and $2-\mu \mathrm{m}$-depth template) the roughness presents a maximum and at even number (the case of $0.75-\mu \mathrm{m}, 1.25-\mu \mathrm{m}, 1.75-\mu \mathrm{m}$ depth template) the roughness improves. This is due to the second laser pass which improves the roughness that the previous laser pass pattern.

As shown in Figure 7, the width of the channel has higher accuracy when the channel width and depth are lower. Results also denote that channel depth has a higher influence on accuracy. For channels with less than $1 \mu \mathrm{m}$-depth, the deviation is between $0.4 \%$ and the deviation reaches $7 \%$ when the channel width increases. The accuracy regarding the depth increases with it reaching a maximum of $10 \%$ deviation for the $2 \mu \mathrm{m}$-depth channels. As it is mentioned before, to achieve higher depth, the number of laser passes increases. This fact has a certain effect on the distance between the columns defined. This distance increases $550 \mathrm{~nm} \pm 100 \mathrm{~nm}$ at each laser pass from the designed dimensions. This accuracy is one order of magnitude higher than in conventional soft lithography. However, the depth of the channels is unachievable by soft lithography. On the other hand, when the channel depth increases, the channel width increases as well, therefore, lowering the precision, but being still similar to the soft lithography process. For wider channels, the accuracy of the laser decreases exponentially as shown in Figure 8 . This figure shows the case of the template of $30 \mu \mathrm{m}$ width and $0.75 \mu \mathrm{m}$ depth, where the deviation is $1.54 \mu \mathrm{m}$ and this deviation increases with the depth reaching a $4.31 \mu \mathrm{m}$ deviation for the template of $2 \mu \mathrm{m}$-depth. As it is expected, the width of the column has the opposite behavior, as the depth of the channel increases the column becomes narrower and the channel width

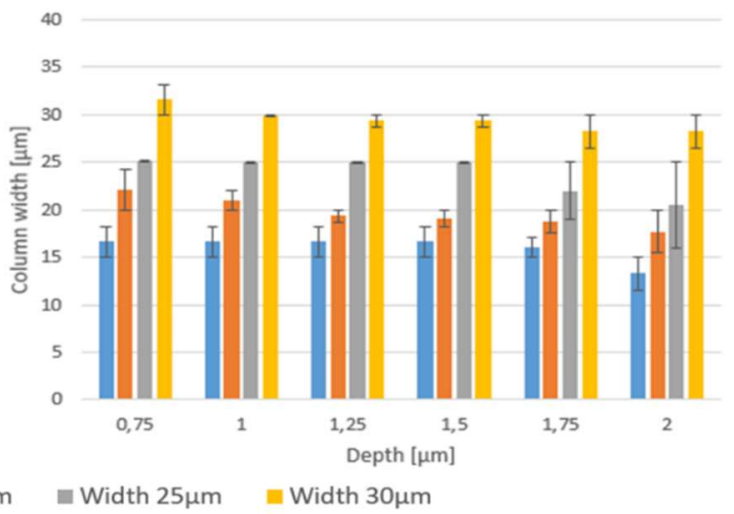

Figure 7: Confocal 3D measurement of the channel width and the column width at the channel template depth $0.75 \mu \mathrm{m}$, $1 \mu \mathrm{m}, 1.25 \mu \mathrm{m}, 1.5 \mu \mathrm{m}, 1.75 \mu \mathrm{m}$, and $2 \mu \mathrm{m}$. 


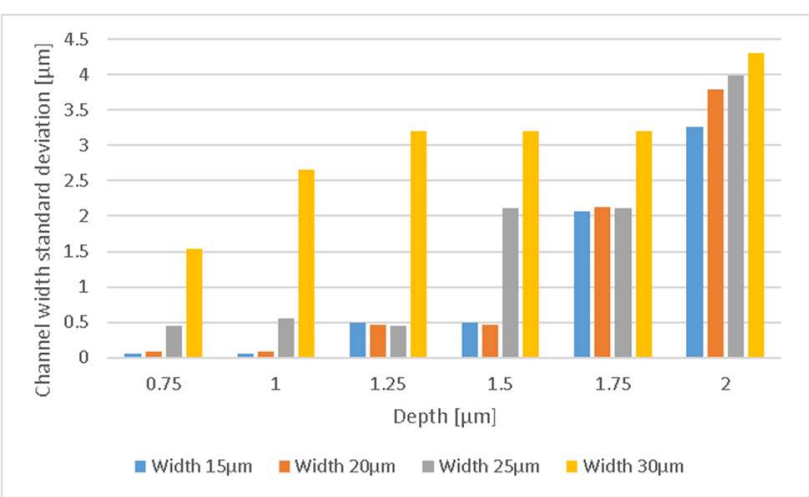

Figure 8: Channel width standard deviation at depth of 0.75 ,

$1,1.25,1.5,1.75$, and $2 \mu \mathrm{m}$.

increases. When the channel depth is low, the width of the column is in an average of a $10 \%$ wider than the designed dimensions and as the channel depth increases this tendency

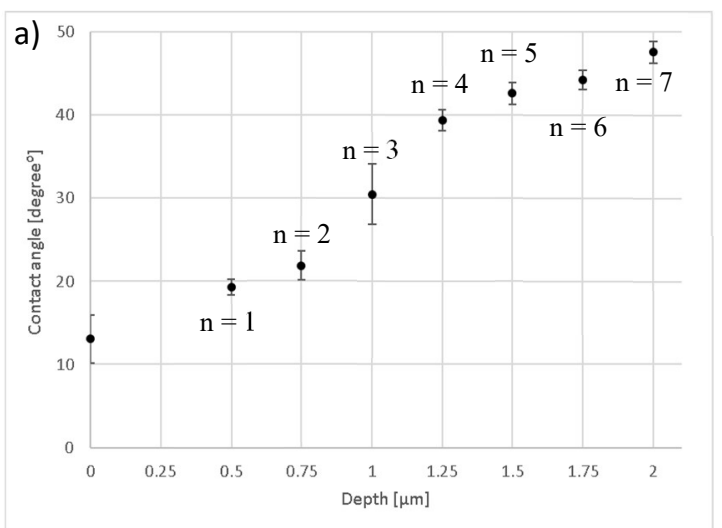

that the contact angle measurements depend mostly on surface texture rather than the roughness.

According to the contact angles measurements as shown in Figure 9 on the sub-micron range, the channel is completely hydrophilic to perform self-driven fluid test [10] and useful for the blood plasma separator applications.

\subsection{Blood plasma separator Application}

Figure 10 shows the schematic of the top view of the BPS device and also the microscopic picture of the channels. Blood was injected from the inlet and flew through the main channel as shown in Figure 10. Afterward, the plasma was spread through the bottom part. Since the height of the pillars was less than the minimum size of RBCs, all the cells were trapped in the main channel. The pure plasma in the bottom part was

Figure 9: (a)Contact angles measurements at the template depth of $0.5 \mu \mathrm{m}, 0.75 \mu \mathrm{m}, 1 \mu \mathrm{m}, 1.25 \mu \mathrm{m}, 1.5 \mu \mathrm{m}, 1.75$ $\mu \mathrm{m}, 2 \mu \mathrm{m}$. Zero is on the surface of the glass. (b)Microscopic picture of the contact angle measurement of a $0.7 \mu l$ of DI water on the glass template depth of $0 \mu \mathrm{m}$.

decrease; for the case of $2 \mu \mathrm{m}$-depth channels, the width of the column is in an average of a $3.6 \%$ narrower than the designed dimensions.

The angle of contact between pure water and a perfectly clean glass is $0^{\circ}$ and for ordinary water increase from $8^{\circ}$ to $18^{\circ}$. Figure 9a shows the static contact angle on glass samples irradiated at laser pass of $2,3,4,5,6$, and 7 , revealing a hydrophilic behavior for all the different laser passes but show a dependence of the wettability on the laser passes employed. As shown in Figure $9 \mathrm{~b}$ the measured contact angle is $11^{\circ}$, since dionized water is used on a slide of glass.

This actual behavior can be explained assuming that the texture generated during the laser machining, trap air between the water and the glass substrate below, preventing a complete wetting of the substrate. Interferometry images of Figure $4 \mathrm{a}$ and Figure 5a show the cross-section of the profile shape, characterized by laser groove and peaks. The dimension of the grooves does not appear to change significantly, confirming
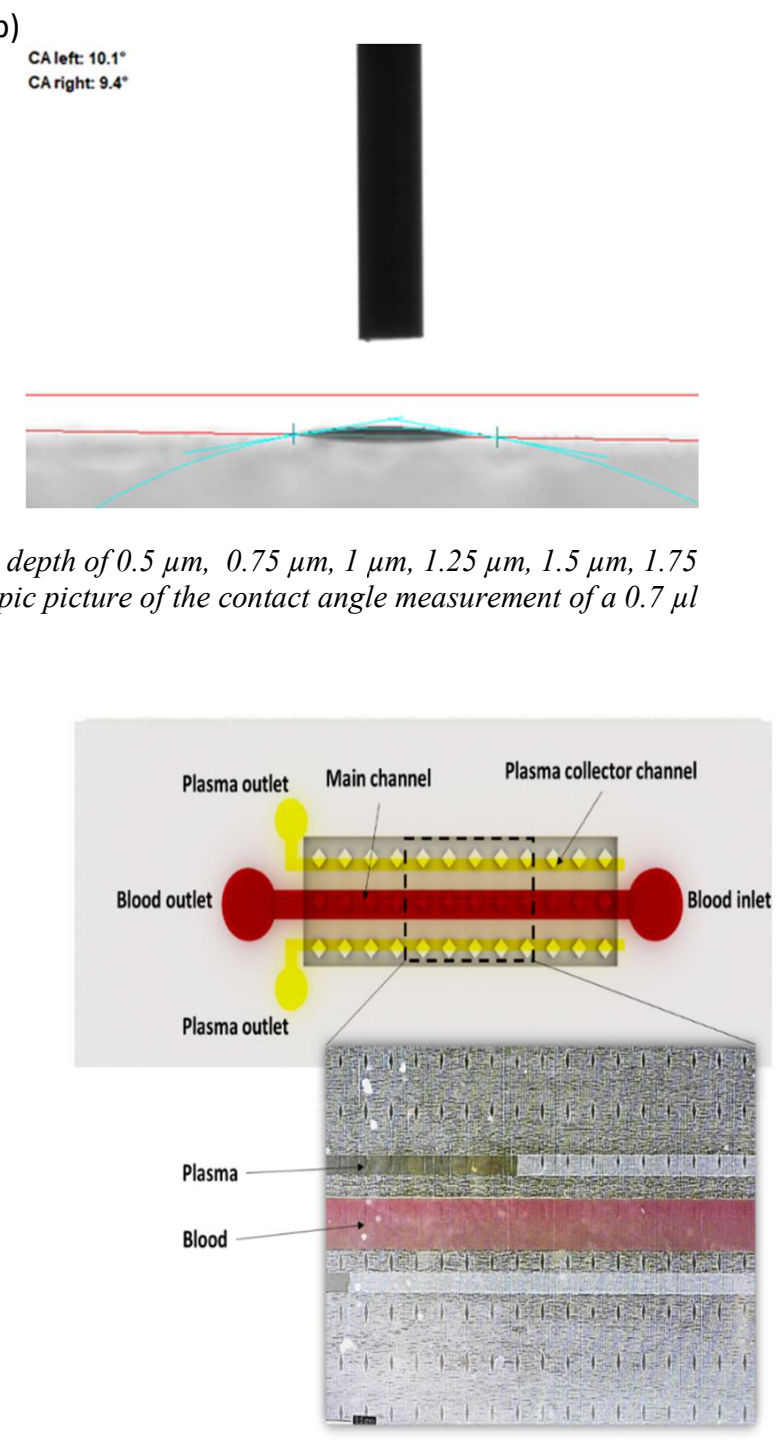

Figure 10: The schematic of BPS device from the top view and the microscopic view of the BPS right after the blood injection. 
filled in two lateral channels by the capillary. Figure 10 also shows the filling process of plasma into the lateral channels. After directly counting the number of blood cells in blood inlet and plasma outlets, by the help of ImageJ software, it has been confirmed that final blood cells in the plasma collector channels were zero. Since no cell could penetrate to the lateral channel, the purity of the extracted plasma according to equation 1 was obtained $100 \%$. The excellent purity of the extracted plasma is the significant advantage of this BPS. This figure also denotes the high transparency of the BPS that allows analyzing the calorimetry and particle counting.

\section{Conclusion}

A novel method to manufacture a watertight sub-micron channel has been developed and tested. The open-top submicron channels with an array of pillars were manufactured using a femtosecond laser and the watertight property was reached by a PDMS cover. The femtosecond laser has been evaluated to manufacture sub-micron channels above $500 \mathrm{~nm}$. The applicability of the manufactured sub-micron channels have been validated in a BPS device. Plasma was successfully extracted from blood with the highest efficiency of $100 \%$. This method has several advantages compared to current submicron watertight manufacturing methods:

- It is a rapid manufacturing method for channel dimension greater than $750 \mathrm{~nm}$ in depth. (about 40 seconds for a rectangles with the area of $18.5 * 12.5 \mathrm{~mm}^{2}$ )

- The PDMS is not sticking to the sub-micron channel either during the bonding fabrication or operating the device due to the texture of the surface.

- The number of laser passes has a significant influence on the pattern. The odd number of laser pass increases the roughness while the next laser pass decreases the roughness.

- The number of laser passes has an influence on the contact angles: as the laser passes increase, the surface wettability decreases.

- The channel width precision decreases when increasing the channel width and depth.

- Laser focus spot size limits the decrease of the width of the sub-micron channel.

- The glass maintains good transparency and enables colorimetric and particle counts analysis systems.

\section{Acknowledgments}

This work is partially supported by Microrelleus, S.L (www.microrelleus.com) and the Spanish Ministry of Economy and Competitivity, grant nos. CTQ2016-77936-R (funding also from FEDER) and CTQ2017-84966-C2-1R. Special acknowledgment to Raúl García from Microrelleus, S.L for his support on the laser manufacturing.

\section{References}

[1] Yanazawa, H. and Homma, K., 2017 Growing market of MEMS and technology development in process and tools specialized to MEMS. In 2017 IEEE Electron Devices Technology and Manufacturing Conference (EDTM) (pp. 143144). IEEE.

[2] Laermer, F., 2018 MEMS at Bosch-Invented for life. In 2018 IEEE Micro Electro Mechanical Systems (MEMS) (pp. 237240). IEEE.

[3] De Marco, C., Eaton, S. M., Suriano, R., Turri, S., Levi, M., Ramponi, R., ... \& Osellame, R. 2010 Surface properties of femtosecond laser ablated PMMA. ACS applied materials \& interfaces, 2(8), 2377-2384.

[4] Yoon, Y., Lee, J., Ra, M., Gwon, H., Lee, S., Kim, M.Y., Yoo, K.C., Sul, O., Kim, C.G., Kim, W.Y. and Park, J.G., 2019 Continuous Separation of Circulating Tumor Cells from Whole Blood Using a Slanted Weir Microfluidic Device. Cancers, 11(2), p.200.

[5] Patel, J.N., Gray, B.L., Kaminska, B., Wu, N.C. and Gates, B.D., 2013 SU-8-and PDMS-based hybrid fabrication technology for combination of permanently bonded flexible and rigid features on a single device. Journal of Micromechanics and Microengineering, 23(6), p.065029.

[6] Mehrdel, P., Karimi, S., Farré-Lladós, J. and Casals-Terré, J., 2018 Novel Variable Radius Spiral-Shaped Micromixer: From Numerical Analysis to Experimental Validation. Micromachines, 9(11), p.552.

[7] Chhina, S.K., Perez, C.F. and Parameswaran, M., 2012 Microfluidic system to detect DNA amplicons using agglutination technique. Journal of Micromechanics and Microengineering, 22(11), p.115038.

[8] Farré-Lladós, J., Westerberg, L.G. and Casals-Terré, J., 2017 New method for lubricating wind turbine pitch gears using embedded micro-nozzles. Journal of mechanical science and technology, 31(2), pp.797-806.

[9] Mackenzie, M.D. and Kar, A.K., 2019 Microfluidic devices and biological lasers for biophotonic applications. In Journal of Physics: Conference Series (Vol. 1151, No. 1, p. 012001). IOP Publishing.

[10] Madadi, H., Casals-Terré, J., Castilla-López, R. and SuredaAnfres, M., 2014 High-throughput microcapillary pump with efficient integrated low aspect ratio micropillars. Microfluidics and nanofluidics, 17(1), pp.115-130.

[11] Madadi, H., Casals-Terré, J. and Mohammadi, M., 2015 Selfdriven filter-based blood plasma separator microfluidic chip for point-of-care testing. Biofabrication, 7(2), p.025007.

[12] Sutera, S.P. and Mehrjardi, M.H., 1975 Deformation and fragmentation of human red blood cells in turbulent shear flow. Biophysical journal, 15(1), pp.1-10. 
[13] Trotta, G., Volpe, A., Ancona, A. and Fassi, I., 2018 Flexible micro manufacturing platform for the fabrication of PMMA microfluidic devices. Journal of Manufacturing Processes, 35, pp.107-117.

[14] Chen, L., Wen, J., Zhang, P., Yu, B., Chen, C., Ma, T., Lu, X. Kim, S.H. and Qian, L., 2018 Nanomanufacturing of silicon surface with a single atomic layer precision via mechanochemical reactions. Nature communications, 9(1), p.1542.

[15] Karimi, S., Farré-Lladós, J., Mir, E., Escolar, G. and CasalsTerré, J., 2019 Hemostasis-On-a-Chip: Impedance Spectroscopy Meets Microfluidics for Hemostasis Evaluation. Micromachines, 10(8), p.534.

[16] Chan, Y.C., Lee, Y.K. and Zohar, Y., 2006 High-throughput design and fabrication of an integrated microsystem with high aspect-ratio sub-micron pillar arrays for free-solution micro capillary electrophoresis. Journal of micromechanics and microengineering, 16(4), p.699.

[17] Wu, Z., Yan, H., Chen, H. and Huang, H., 2009 One-stage fabrication of sub-micron hydrophilic microchannels on PDMS. Applied Surface Science, 255(8), pp.4702-4704.

[18] Chen, X. and Zhang, L., 2018 Review in manufacturing methods of nanochannels of bio-nanofluidic chips. Sensors and Actuators B: Chemical, 254, pp.648-659.

[19] Park, S.M., Huh, Y.S., Craighead, H.G. and Erickson, D., 2009 A method for nanofluidic device prototyping using elastomeric collapse. Proceedings of the National Academy of Sciences, 106(37), pp.15549-15554.

[20] Bu, M., Melvin, T., Ensell, G.J., Wilkinson, J.S. and Evans, A.G., 2004 A new masking technology for deep glass etching and its microfluidic application. Sensors and Actuators A: Physical, 115(2-3), pp.476-482.

[21] Garra, J., Long, T., Currie, J., Schneider, T., White, R. and Paranjape, M., 2002 Dry etching of polydimethylsiloxane for microfluidic systems. Journal of Vacuum Science \& Technology A: Vacuum, Surfaces, and Films, 20(3), pp.975-982.

[22] Chryssolouris, G., 2013 Laser machining: theory and practice. Springer Science \& Business Media.

[23] Liu, H.B. and Gong, H.Q., 2009 Templateless prototyping of polydimethylsiloxane microfluidic structures using a pulsed $\mathrm{CO} 2$ laser. Journal of Micromechanics and Microengineering, 19(3), p.037002.

[24] Liu, K., Xiang, J., Ai, Z., Zhang, S., Fang, Y., Chen, T., Zhou, Q., Li, S., Wang, S. and Zhang, N., 2017 PMMA microfluidic chip fabrication using laser ablation and low temperature bonding with OCA film and LOCA. Microsystem Technologies, 23(6), pp.1937-1942.

[25] Wang, Z.K., Zheng, H.Y., Lim, C.P. and Lam, Y.C., 2009 Polymer hydrophilicity and hydrophobicity induced by femtosecond laser direct irradiation. Applied physics letters, 95(11), p.111110.

[26] Mohammed, M.I., Quayle, K., Alexander, R., Doeven, E., Nai, R., Haswell, S.J., Kouzani, A.Z. and Gibson, I., 2015 Improved manufacturing quality and bonding of laser machined microfluidic systems. Procedia Technology, 20, pp.219-224.

[27] Roberts, M.A., Rossier, J.S., Bercier, P. and Girault, H., 1997 UV laser machined polymer substrates for the development of microdiagnostic systems. Analytical chemistry, 69(11), pp.20352042.
[28] Ben-Yakar, A. and Byer, R.L., 2004 Femtosecond laser ablation properties of borosilicate glass. Journal of applied physics, 96(9), pp.5316-5323.

[29] Jagannadh, V.K., Mackenzie, M.D., Pal, P., Kar, A.K. and Gorthi, S.S., 2014 Imaging flow cytometry with femtosecond laser-micromachined glass microfluidic channels. IEEE Journal of Selected Topics in Quantum Electronics, 21(4), pp.370-375.

[30] Mastrangelo, C.H., 1999 Suppression of stiction in MEMS. MRS Online Proceedings Library Archive, 605.

[31] Wenzel, R.N., 1936 Resistance of solid surfaces to wetting by water. Industrial \& Engineering Chemistry, 28(8), pp.988-994.

[32] Cassie, A.B.D. and Baxter, S., 1944 Wettability of porous surfaces. Transactions of the Faraday society, 40, pp.546-551. 